\title{
Identifying risk factors: Challenges of separating signal from noise
}

\author{
Jeevanantham Rajeswaran, $\mathrm{PhD},{ }^{\mathrm{a}}$ and Eugene $\mathrm{H}$. Blackstone, $\mathrm{MD}^{\mathrm{a}, \mathrm{b}}$
}

\footnotetext{
From the Departments of ${ }^{\mathrm{a}}$ Quantitative Health Sciences and ${ }^{\mathrm{b}}$ Thoracic and Cardiovascular Surgery, Cleveland Clinic, Cleveland, Ohio.

Funding: Cleveland Clinic.

Disclosures: Authors have nothing to disclose with regard to commercial support.

Received for publication Jan 12, 2017; accepted for publication Jan 26, 2017; available ahead of print Feb 22, 2017.

Address for reprints: Eugene H. Blackstone, MD, Department of Thoracic and Cardiovascular Surgery, Cleveland Clinic, 9500 Euclid Ave, Desk JJ-4, Cleveland, OH 44195 (E-mail: blackse@ ccf.org).

J Thorac Cardiovasc Surg 2017;153:1136-8

$0022-5223 / \$ 36.00$

Copyright $(c) 2017$ by The American Association for Thoracic Surgery

http://dx.doi.org/10.1016/j.jtcvs.2017.01.010
}

Identifying risk factors from a possibly large number of candidate variables - variable selection-is key in clinical outcomes studies. However, different persons analyzing the same dataset often identify different risk factors. ${ }^{1}$ Making variable selection more reproducible is the challenge addressed by Karim and colleagues in this issue of the Journal. ${ }^{2}$

\section{BAGGING PREDICTORS ${ }^{3}$}

The approach taken by Karim and colleagues is based on resampling (Figure 1). ${ }^{4}$ Observations in a dataset of size $n$ are selected randomly with replacement to populate a new analysis dataset of equal size (a bootstrap sample). Many such datasets are generated, and each is analyzed. $^{3,5}$ It is rare that the identical risk factors are found, ${ }^{6}$ confirming the suspicion that analysis of a single dataset produces unstable (unreproducible) results. However, if one aggregates results of these many analyses, signal separates from noise. A macro library is available from the authors for regression procedures in SAS (SAS Institute, Cary, NC).

\section{PREPARING THE DATASET FOR ANALYSIS}

Not discussed by Karim and colleagues are some challenges of preparing the dataset for such an analysis, because one does not want to repeat 1000 analyses due to a mistake!

\section{Missing Data}

Screen all covariables and discard those with a large amount of data missing. How much is too much depends on the degree of redundancy within available variables; perhaps $25 \%$ is reasonable for conventional missing data methods. For remaining variables having missing data, statistically acceptable approaches include multiple imputation, $^{7}$ as used by Karim and colleagues, although interesting new machine-learning methods are being developed. Analyzing only patients with complete data for all variables is generally unacceptable.

\section{Sufficient Data} consideration. (Figure 2). ${ }^{10,11}$

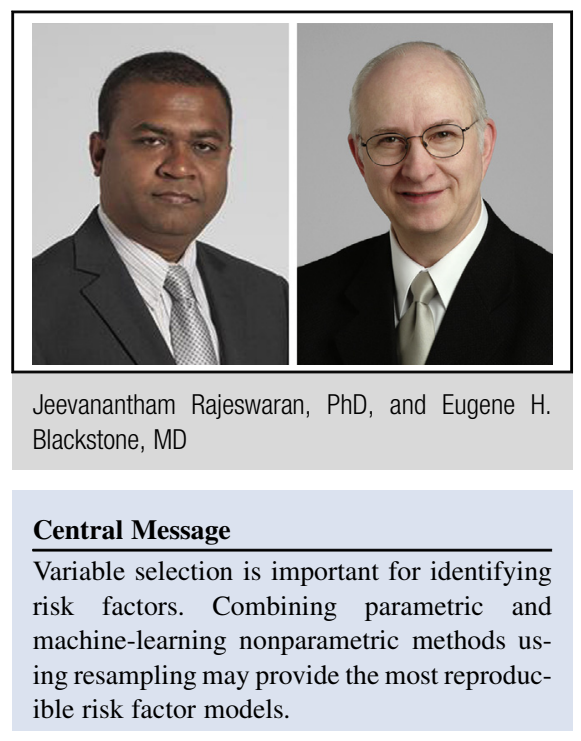

See Article page 1128 .

Screen categorical covariables in relationship to categorical endpoints, such as death. The effective sample size for such analyses is number of observed events. When few endpoints are available for a categorical variable (5-10 or less), any analysis with such a variable will be unstable, and it should be eliminated from

\section{Continuous Variables}

Use continuous variables as such in models, rather than arbitrarily categorizing them. Recognize, however, that a continuous variable may have a nonlinear relationship with the endpoint. After sensitivity analysis, Karim and colleagues decided to use no transformations of scale for continuous variables. However, the main reason for using transformations or spline representations for continuous variables is to meet model assumptions. For example, logistic regression assumes that continuous variables are associated linearly with the logit of the binary response. Alternatively, the multivariable analysis can be supplemented by a completely nonparametric analysis, eg, random forests ${ }^{9}$; partial dependency plots will illuminate the risk-adjusted shape of a continuous variable vis-a-vis outcome to guide model development 

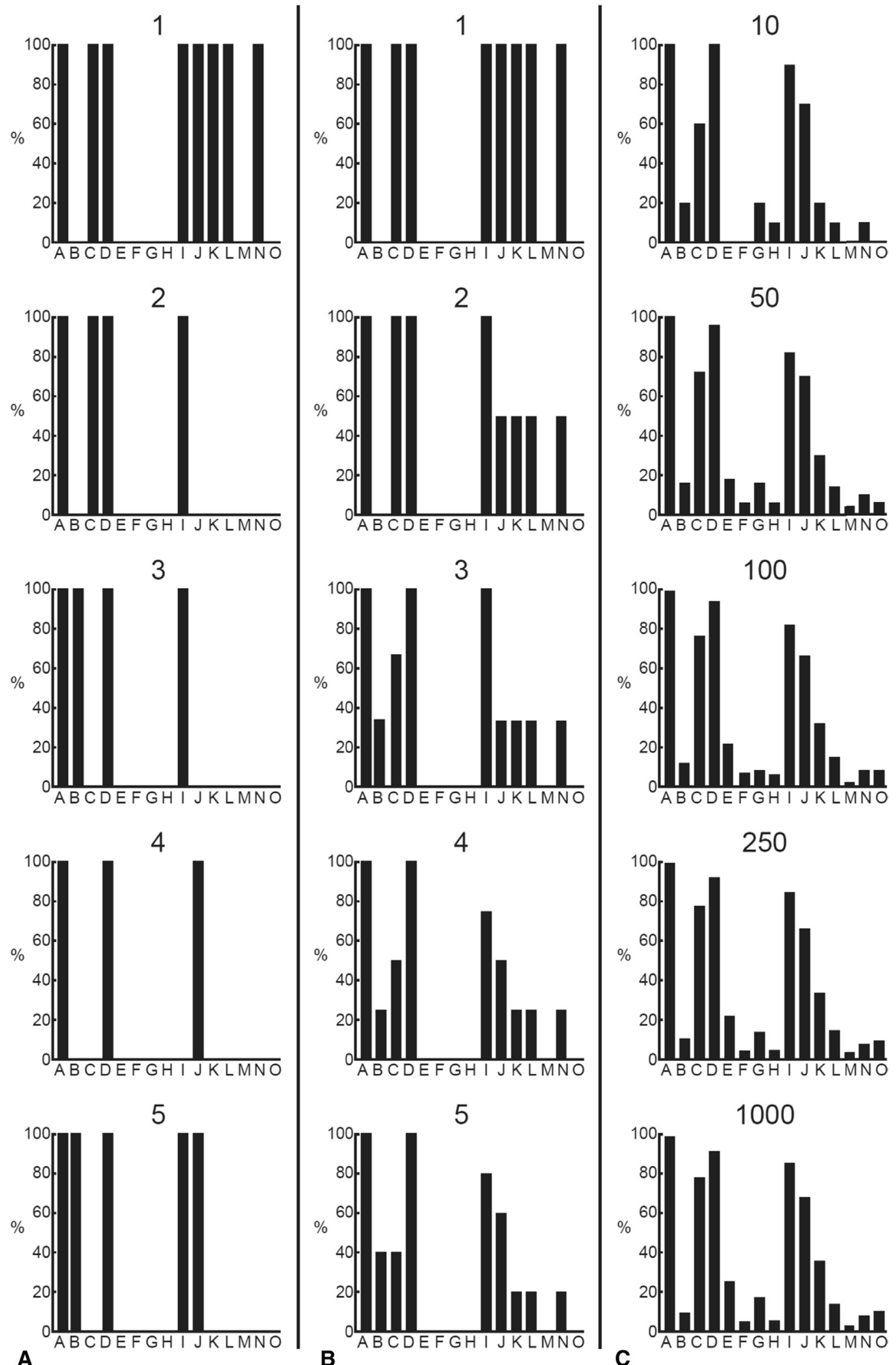

FIGURE 1. Example of automated variable selection by bootstrap aggregation (bagging), depicting the advantage of averaging. Fifteen variables labeled A through $\mathrm{O}$ are depicted as potential predictors of death after mitral valve surgery. In A, analyses of 5 bootstrap samples are shown. Tall bars indicate the variable was selected at $P<.05$, and gaps represent variables not selected. B and C show running averages as the bootstrap samples increased from 1 to 1000. Notice that no variable was selected $100 \%$ of the time, and all 15 were selected at one time or another. From Blackstone and colleagues. ${ }^{4}$ 


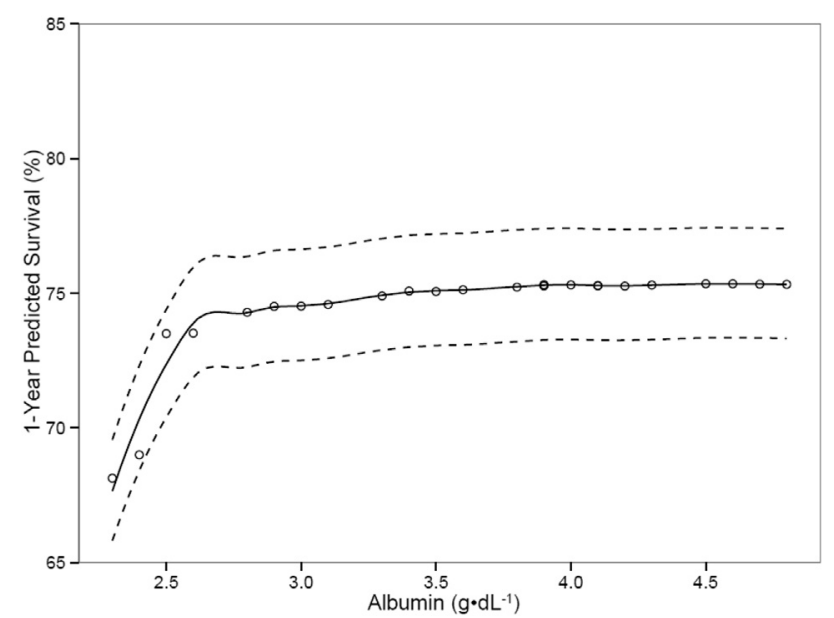

FIGURE 2. Random forest for survival partial dependency plot depicts the nonlinear relationship between albumin and 1-year predicted survival. ${ }^{10}$ Graph presents a risk-adjusted estimate of the effect of albumin on survival after aortic valve replacement. Circles and solid lines represent risk-adjusted estimates, and dashed lines indicate $68 \%$ confidence band. From Szeto and colleagues. ${ }^{11}$

\section{Selecting Candidate Variables for Multivariable Analysis}

The final step in preparing for analysis is to compile the list of candidate variables. Karim and colleagues used expert opinion and current literature to compile "plausible predictors," an approach that may preclude addition of new knowledge. Others use $P$ values based on a series of univariable models, selecting candidates that are statististically significant. This approach ignores lurking variables, ie, variables that are not statistically significant until another variable is taken into account. ${ }^{12,13}$ We recommend incorporating all available and well-vetted variables in model building, coupled with full exploitation of the machine-learning aspect of bootstrap methods that can efficiently and effectively identify the most reliable variables (signal).

\section{VARIABLE SELECTION PROCESS}

The result of analyzing data may depend on the variable selection strategy, which is explored by Karim and colleagues. We only caution that backward selection starts with all variables forced into the model, generally requiring a large effective sample size, such as available in the study of Karim and colleagues. This can be achieved in a machine-learning environment by incorporating random subsets of variables instead. ${ }^{10}$

\section{Are We There Yet?}

Not discussed by Karim and colleagues is the matter of colinearity—high correlation among variables. Examples are creatinine, blood urea nitrogen, and estimated glomerular filtration rate; height, weight, and body surface area; and multiple candidate transformations of scale for continuous variables. Thus, another step is clustering of highly correlated variables. After bagging is completed, calculate how often the cluster of variables has been selected. The most commonly selected or informative representative of the cluster may be identified as a risk factor. This same counting process will indicate how many terms are needed to represent a nonlinear relationship of a continuous variable.

\section{ALTERNATIVES}

Karim and colleagues note in their Discussion that machine-learning approaches open up new methods, ${ }^{14}$ such as random forests, ${ }^{9,10}$ for identifying important variables related to outcome without need for $P$ values, superior ways to model continuous variables, and ways to account for complex interelations among variables. These methods, being rapidly developed and used by the Microsofts, Amazons, and Googles of the world, will, we believe, replace many traditional statistical methods ${ }^{15}$ and provide better predictive models, yielding superior insight into the data.

\section{References}

1. Naftel DC. Do different investigators sometimes produce different multivariable equations from the same data? J Thorac Cardiovasc Surg. 1994;107:1528-9.

2. Karim MN, Reid CM, Tran L, Cochrane A, Billah B. Variable selection methods for multiple regressions influence the parsimony of risk prediction models for cardiac surgery. J Thorac Cardiovasc Surg. 2017;153:1128-35.e3.

3. Breiman L. Bagging predictors. Machine Learning. 1996;24:123-40.

4. Blackstone EH, Lenat DB, Ishwaran H. Methods that need to be developed. In: Institute of Medicine. Learning What Works: Infrastructure Required for Comparative Effectiveness Research—Workshop Summary. Washington, DC: National Academies Press; 2011:123-43.

5. Sauerbrei W, Schumacher M. A bootstrap resampling procedure for model building: application to the Cox regression model. Stat Med. 1992;11:2093-109.

6. Diaconis P, Efron B. Computer-intensive methods in statistics. Sci Am. 1983;248: 116-30.

7. Rubin DB. Multiple Imputation for Non-Response in Surveys. New York: Wiley; 1987.

8. Blackstone EH. Sufficient data. J Thorac Cardiovasc Surg. 2016;152:1235-6.

9. Breiman L. Random forests. Machine Learning. 2001;45:5-32.

10. Ishwaran H, Kogalur UB, Blackstone EH, Lauer MS. Random survival forests. Ann Appl Stat. 2008;2:841-60.

11. Szeto WY, Svensson LG, Rajeswaran J, Ehrlinger J, Suri RM, Smith CR, et al. Appropriate patient selection or health care rationing? Lessons from surgical aortic valve replacement in the Placement of Aortic Transcatheter Valves I trial. J Thorac Cardiovasc Surg. 2015;150:557-68.e11.

12. Joiner BL. Lurking variables: some examples. Am Stat. 1981;35:227.

13. Gum PA, Thamilarasan M, Watanabe J, Blackstone EH, Lauer MS. Aspirin use and all-cause mortality among patients being evaluated for known or suspected coronary artery disease: a propensity analysis. Jama. 2001;286: 1187-94.

14. Iswaran H. Variable importance in binary regression trees and forests. Electron J Statist. 2007; 1:519-37.

15. Breiman L. Statistical modeling: the two cultures. Statist Sci. 2001;16: 199-231. 